\title{
USING ECMO VV IN THE COVID-19 PANDEMIC
}

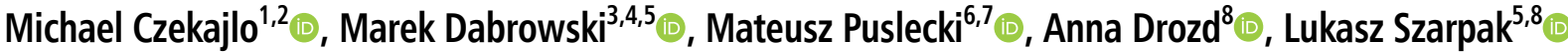 \\ ${ }^{1}$ Healthcare Innovation, Hunter Holmes McGuire VA Medical Center, Richmond, VA, USA \\ ${ }^{2}$ Department of Anesthesiology, Virginia Commonwealth University, Richmond, VA, USA \\ ${ }^{3}$ Chair and Department of Medical Education, Poznan University of Medical Sciences, Poznan, Poland \\ ${ }^{4}$ Polish Society of Medical Simulation, Poland \\ ${ }^{5}$ Polish Society of Disaster Medicine, Warsaw, Poland \\ ${ }^{6}$ Department of Medical Rescue, Chair of Emergency Medicine, Poznan University of Medical Sciences, Poznan, Poland \\ ${ }^{7}$ Department of Cardiac Surgery and Transplantology, Poznan University of Medical Sciences, Poznan, Poland \\ ${ }^{8}$ Lazarski University, Warsaw, Poland
}

KEY WORDS: ecmo, COVID-19, VV

Disaster Emerg Med J 2020; 5(2)

The procedure for the techniques of extracorporeal support of vital functions (Extracorporeal Membrane Oxygenation) can be an effective therapeutic tool for critically ill patients [1]. The application of this method within the COVID-19 pandemic, however, is only possible with adequate medical personnel and equipment [2]. COVID-19 is a disease caused by the new SARS-CoV-2 virus that appeared in December 2019 and has now become a worldwide pandemic with rapid spread [3]. On April 21, there were 2.497.000 confirmed cases with 656.051 recovered patients and, unfortunately, 171.245 deaths. In Poland, which has more than 38 million inhabitants, there were 9.737 confirmed cases and 385 deaths, and the USA with 328 million inhabitants with confirmed 793.000 cases, 42.518 deaths, and 72.390 recovered patients [4-6]. The spectrum of the SARSCoV-2 associated pneumonia is mild to life-threatening critical situations and requiring critical treatment. Reports indicate that most COVID-19 patients have moderate symptoms (fever, cough and fatigue) and recover quickly, however, group of patients develop severe respiratory failure and acute respiratory distress syndrome (ARDS) necessitating intensive care. Unfortunately, the death rate of COVID-19 requiring mechanical ventilation is high. ECMO can be a lifesaving measure for patients with severe ARDS or refractory cardiovascular disease [7-9].

ECMO is a method of extracorporeal membrane blood oxygenation implemented via an oxygenator responsible for gas exchange and pumps for blood circulation. Depending on the technique, the device is connected to the patient's vessels, and used to support the insufficient respiratory system - ECMO $\mathrm{W}$ venous-venous or the insufficient circulatory and respiratory systems - ECMO VA - venous-arterial. The ECMO is not treatment but support technique, it offers the prospect of prolonging the time of therapy and regeneration of insufficient systems $[10,11]$.

ECMO is an invasive therapy with a high risk of sudden and severe life-threatening complications (disconnection of the system, dislocation of cannulas, air embolism). For this reason, a constant presence of at least two medical personnel trained in ECMO therapy is necessary. When planning the care for a patient with suspected or confirmed infection with the SARS-CoV-2 virus, the balance between the potential benefits of ECMO and the available personnel (with appropriate experience and training) as well as hospital equipment (including personal protective equipment) and infrastructure should be explicitly secure $[2,8,12]$. The risk to medical personnel and the need to care for other critically ill patients should also be taken into account. This is particularly essential, as it becomes challenging to work in complicated and advanced PPE for the personnel of Intensive Care Units, who are required to spend prolonged periods with a patient connected to the ECMO device [13].

In critical patients, mechanical ventilation is the primary method of supportive therapy. In the case of 
SARS, a vital element of the procedure is additionally the low-pressure ventilation schemes with low tidal volume ventilation $(\mathrm{Vt})(\mathrm{Vt} 4-8 \mathrm{ml} / \mathrm{kg}$ of predicted body weight) and pressure plateau orientation (Pplat) $<30 \mathrm{~cm} \mathrm{H2O}$ and a higher positive end pressure strategy exhaust. This is utilized to minimize lung damage caused by respiratory therapy [14]. To minimize the potential for lung damage, and when there is a lack of available ventilation equipment, ECMO becomes useful in the treatment of COVID-19 patients [15]. The ELSO report shows that during the advancement of the pandemic there was an increase in the use of the ECMO therapy, and at the time of writing this letter, 901 patients were supported by ECMO (average age is 52 years, $95 \% \mathrm{VV}$ ECMO, 5\% VA ECMO and other configurations) [3].

Currently, in Poland, five centers have been selected to meet the challenges of using ECMO VV for COVID-19 patients. The application of ECMO therapy during the COVID-19 pandemic is challenging yet achievable, offering a high chance of patient survival.

\section{REFERENCES:}

1. Goligher EC, Tomlinson G, Hajage D, et al. EOLIA Trial Group, REVA, and ECMONet. Extracorporeal Membrane Oxygenation for Severe Acute Respiratory Distress Syndrome. N Engl J Med. 2018; 378(21): 1965-1975, doi: 10.1056/NEJMoa1800385, indexed in Pubmed: 29791822.

2. Smereka J, Puslecki M, Ruetzler $\mathrm{K}$, et al. Extracorporeal membrane oxygenation in COVID-19. Cardiol J. 2020 [Epub ahead of print], doi: 10.5603/CJ.a2020.0053, indexed in Pubmed: 32285929.

3. ECMO-Extracorporeal Life Support in Adults. 2014, doi: 10.1007/97888-470-5427-1.
4. https://www.elso.org/Portals/0/Files/pdf/ELSO\%20covid\%20guidelines\%20final.pdf, 21.04.2020.

5. www.populationof.net, 21.04.2020.

6. www.worldometers.info, 21.04.2020.

7. www.census.gov, 21.04.2020.

8. Huang $C$, Wang $Y, L i X$, et al. Clinical features of patients infected with 2019 novel coronavirus in Wuhan, China. Lancet. 2020; 395(10223): 497-506, doi: 10.1016/50140-6736(20)30183-5, indexed in Pubmed: 31986264.

9. Ruetzler K, Szarpak L, Filipiak K, et al. The COVID-19 pandemic - a view of the current state of the problem. Disaster and Emergency Medicine Journal. 2020, doi: 10.5603/demj.a2020.0015.

10. Smereka J, Szarpak L. COVID 19 a challenge for emergency medicine and every health care professional. Am J Emerg Med. 2020 [Epub ahead of print], doi: 10.1016/j.ajem.2020.03.038, indexed in Pubmed: 32241630.

11. Puślecki $M$, Ligowski $M$, Dąbrowski $M$, et al. BEST Life-"Bringing ECMO Simulation To Life"-How Medical Simulation Improved a Regional ECMO Program. Artif Organs. 2018; 42(11): 1052-1061, doi: 10.1111/ aor.13332, indexed in Pubmed: 30043501.

12. www.ecmo.pl, 20.04.2020.

13. Smereka J, Szarpak L, Filipiak K. Modern medicine in COVID-19 era. Disaster and Emergency Medicine Journal. 2020, doi: 10.5603/demj. a2020.0012.

14. Mossadegh C. Monitoring the ECMO. W: Mossadegh C, Combes A (red). Nursing Care and ECMO. Cham: Springer. ; 2017: 45-70.

15. Thompson BT, Chambers RC, Liu KD. Acute Respiratory Distress Syndrome. N Engl J Med. 2017; 377(6): 562-572, doi: 10.1056/ NEJMra1608077, indexed in Pubmed: 28792873.

16. Yang $X, Y u Y, X u J$, et al. Clinical course and outcomes of critically ill patients with SARS-CoV-2 pneumonia in Wuhan, China: a single-centered, retrospective, observational study. Lancet Respir Med. 2020 [Epub ahead of print], doi: 10.1016/S2213-2600(20)30079-5, indexed in Pubmed: 32105632. 